\title{
Amplified spontaneous emission pulses for high-power supercontinuum generation
}

\author{
Huan Huan Liu, Kin Kee Chow \\ School of Electrical and Electronic Engineering, Nanyang Technological University, 50 Nanyang Avenue, \\ Singapore 639798, Singapore \\ E-mail: kkchow@ntu.edu.sg
}

Published in The Journal of Engineering; Received on 15th January 2016; Accepted on 18th January 2016

\begin{abstract}
The authors demonstrate an incoherent light source based on a reflective semiconductor optical amplifier as pump for high-power supercontinuum generation for the first time. The obtained power level is about $160 \mathrm{~mW}$ and $20 \mathrm{~dB}$ spectral bandwidth is around $170 \mathrm{~nm}$.
\end{abstract}

\section{Introduction}

Supercontinuum (SC) generation has attracted much attention due to a broad range of applications including optical communication, optical coherent tomography, and optical frequency metrology [1]. The common method for SC generation is achieved by a piece of highly non-linear medium pumped by ultra-short pulses $[2,3]$. However, it is not straightforward to increase the output power because non-linear effects and dispersion in high-power amplifiers oppose severe obstacles obtaining clean ultra-short pulses at relatively high average power level. Additionally, the generated SC spectrum tends to have significant ripples due to pulse distortion. An alternative approach to achieve high-power and smooth SC spectrum is using continuous-wave light source or amplified spontaneous emission (ASE) source as pump, in which the pump incoherence can aid SC generation and spectral flatness [4, 5]. Previous reports have demonstrated a flat SC spectrum of 956 $\mathrm{nm}$ with high spectral density over $-10 \mathrm{dBm} / \mathrm{nm}$ by using ASE noise source together with an erbium-doped fibre amplifier [6]. The noise source is composed of a $1.55 \mu \mathrm{m}$ ASE source, an electroabsorption modulator and an external electrical pulsed source, which inevitably increase the complexity and cost of the whole system. In this paper, for the first time to our knowledge, a simple and low-cost ASE noise source based on an electrical-driven reflective semiconductor optical amplifier (RSOA) is applied for SC generation. The generated SC spectrum has achieved power level of $160 \mathrm{~mW}$ and $20 \mathrm{~dB}$ spectral bandwidth of $170 \mathrm{~nm}$.

\section{Experimental setup and results}

Fig. 1 shows the experimental setup for SC generation. The used RSOA is housed in a pigtailed coaxial package based on a TO-56 can together with an FC/APC fibre connector, which is compatible to the following fibre links. Such RSOA utilises InP-based buried heterostructure design with a $\sim 1.2 \mu \mathrm{m}$ wide InGaAsP tensile bulk active region. It has high reflectively coating for the rear facet while low reflectivity of $<10^{-5}$ in the front facet. The electrical bandwidth of RSOA is around $1.1 \mathrm{GHz}$ and the saturated output power is about $5 \mathrm{dBm}$. To obtain optical pulses, the RSOA is directly modulated by an electrical pulsed source which is arbitrary waveform generator (AWG: Agilent 33250A). The AWG can generate nearly-square electrical pulses with maximum repetition rate of $80 \mathrm{MHz}$ and shortest pulse duration of $5 \mathrm{~ns}$. To boost up the optical power produced from the RSOA, two-stage amplification is applied. The preamplifier and the second-stage amplifier can enhance the optical power level up to 10 and $23 \mathrm{dBm}$, respectively. A bandpass filter with centre wavelength of $1547 \mathrm{~nm}$ and $3 \mathrm{~dB}$ bandwidth of $40 \mathrm{~nm}$ is used to suppress the ASE noise due to the preamplifier. Two optical isolators are used to protect the RSOA and the second-stage amplifier from the optical reflection-induced damage. Subsequently, the amplified pulsed beam is launched into $1 \mathrm{~km}$ long highly non-linear fibre (HNLF) for spectral broadening. The HNLF has mode field diameter of $3.7 \mu \mathrm{m}$ and non-linear coefficient of $15(\mathrm{~W} \mathrm{~km})^{-1}$ at $1550 \mathrm{~nm}$. The group velocity dispersion coefficient of the HNLF is $-0.23 \mathrm{ps} / \mathrm{nm} / \mathrm{km}$ and the dispersion slope is about $+0.030 \mathrm{ps} / \mathrm{km} / \mathrm{nm}^{2}$ at $1550 \mathrm{~nm}$. The loss of the HNLF is $<0.7 \mathrm{~dB} / \mathrm{km}$. The optical spectrum and the optical pulse trains are recorded by an optical spectral analyser (Ando AQ6317B) with a resolution of $0.01 \mathrm{~nm}$ and a $1.2 \mathrm{GHz}$ photodetector followed by an oscilloscope (Agilent Infiniium 54832B), respectively.

The threshold voltage for RSOA to emit light is about $1.5 \mathrm{~V}$. The output optical power is $8 \mu \mathrm{W}$, and the centre wavelength of spectrum locates at $1573 \mathrm{~nm}$. When the applied voltage is increased, the centre wavelength of spectrum shifts toward shorter wavelength with a slope of $\sim 30.4 \mathrm{~nm} / \mathrm{V}$. To obtain the centre wavelength of spectrum at C-band, the applied voltage is set to be $\sim 2 \mathrm{~V}$ with an output power of $44 \mu \mathrm{W}$. The generated optical spectrum shows centre wavelength of $1540.692 \mathrm{~nm}$ and $20 \mathrm{~dB}$ spectral bandwidth of $\sim 150 \mathrm{~nm}$. Fig. 2 depicts the optical pulse generated from RSOA which has pulse duration of $5.3 \mathrm{~ns}$ with repetition rate of $50 \mathrm{MHz}$. The optical power is further boosted up to $160 \mathrm{~mW}$ by the two-stage amplification. By launching the amplified optical pulses into the HNLF, the optical spectrum is gradually broadened. Fig. 3 shows optical spectra at different power levels. When the input power level is increased from 1 to $160 \mathrm{~mW}$, the $20 \mathrm{~dB}$ bandwidth is increased from 18 to $170 \mathrm{~nm}$; meanwhile, the spectral density is raised from -48 to $-20 \mathrm{dBm} / \mathrm{nm}$. Compared with the method using an ultra-short pulse train for SC generation, the SC spectrum here has good spectral smoothness [2,3]. To understand why the optical spectrum can be broadened by using ASE light as pump, the simulation is further conducted.

\section{Simulation analysis and discussion}

Considering propagation of ASE light along optical fibre with nanosecond time slot, the starting point is how to describe the pulse mathematically. Previous reports show that an ASE light propagating along optical fibre can be treated as a bunch of noise burst [6]. The behind physical mechanism is that the continuous wave in the presence of perturbation tends to break up into pulses due to noise enhancement through modulation instability gain [6]. Moreover, the pulse-to-pulse coherence of the noise burst does not exist unlike the conventional ultra-short pulses. In this calculation, the ASE light has a fine temporal structure with envelope duration of around $5.3 \mathrm{~ns}$. It contains thousands of short pulses with duration of around $300 \mathrm{fs}$ associated with random amplitude and random phase. The incoherence of such bunch of pulses is presented by adding the amplitude of their optical spectra instead of an interference-induced spectral fringe. An SC generation by such 


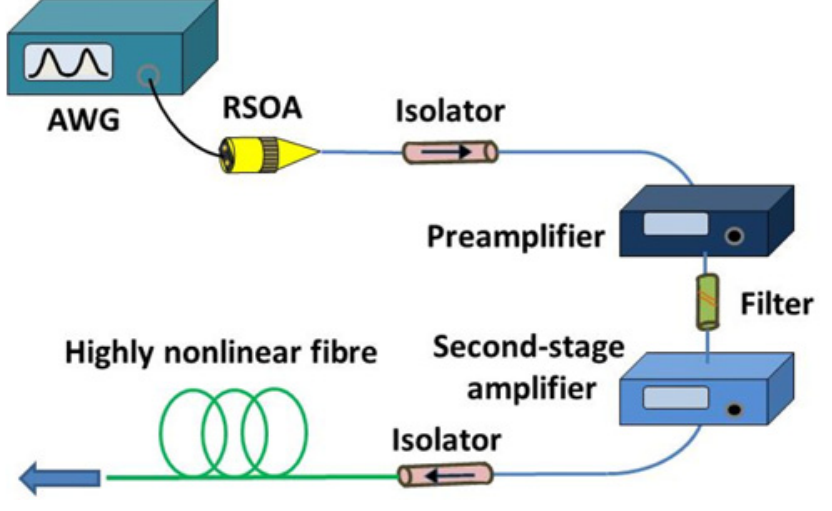

Fig. 1 Experimental setup for SC generation AWG: arbitrary waveform generator and RSOA: reflective semiconductor optical amplifier

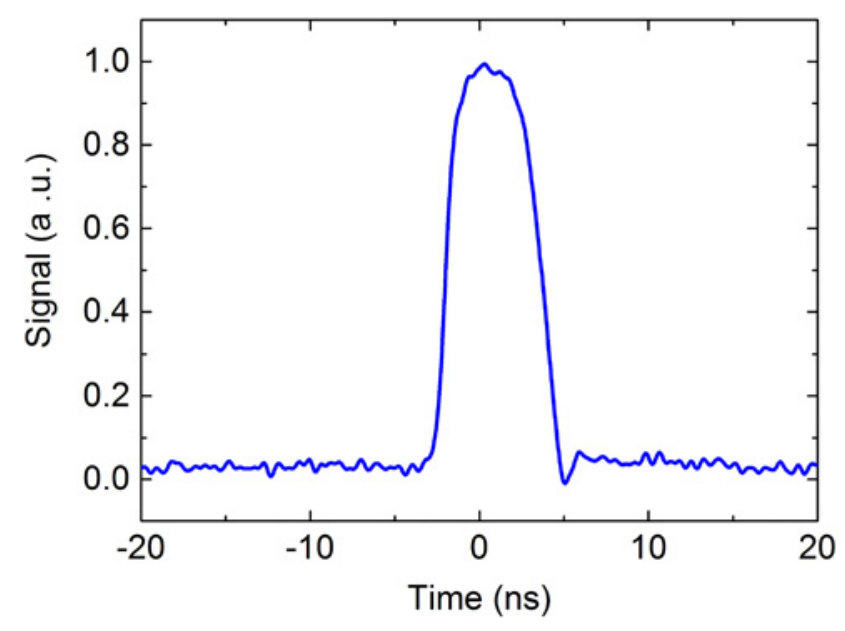

Fig. 2 Optical pulse generation from RSOA

noise burst along optical fibre can be modelled by non-linear Schrodinger equation [7]. The parameter used in the simulation for HNLF is given as follows: fibre non-linearity of $\gamma=15$ (W $\mathrm{km})^{-1}$, group velocity dispersion coefficient of $\beta_{2}=0.23 \mathrm{ps} / \mathrm{nm} /$ $\mathrm{km}$, fibre length of $L=1000 \mathrm{~m}$, the fractional contribution of the delayed Raman-gain spectrum of $f_{\mathrm{R}}=0.18$, and coefficients of Raman-gain spectrum of $\tau_{1}=12.2 \mathrm{fs}$ and $\tau_{2}=32 \mathrm{fs}$.

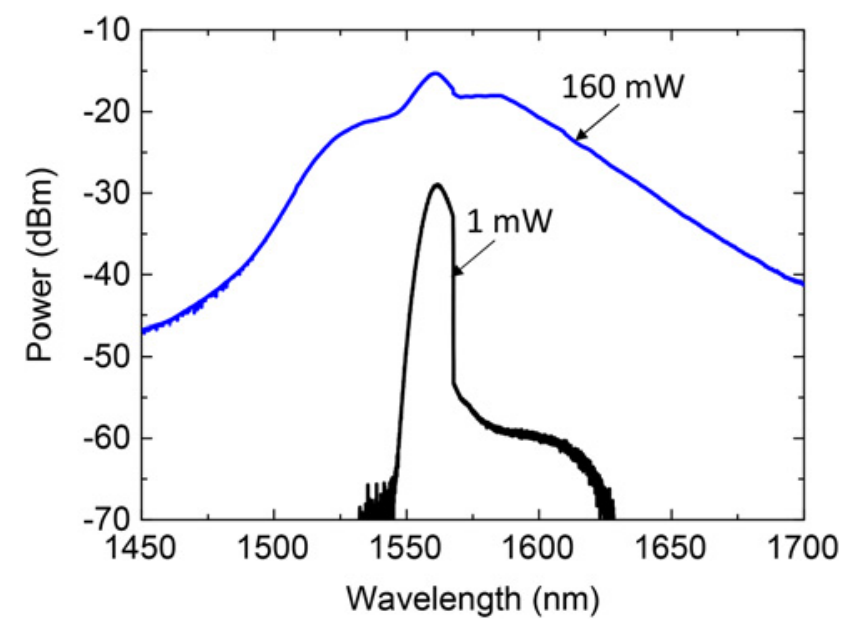

Fig. 3 Spectral broadening in HNLF at various input power levels

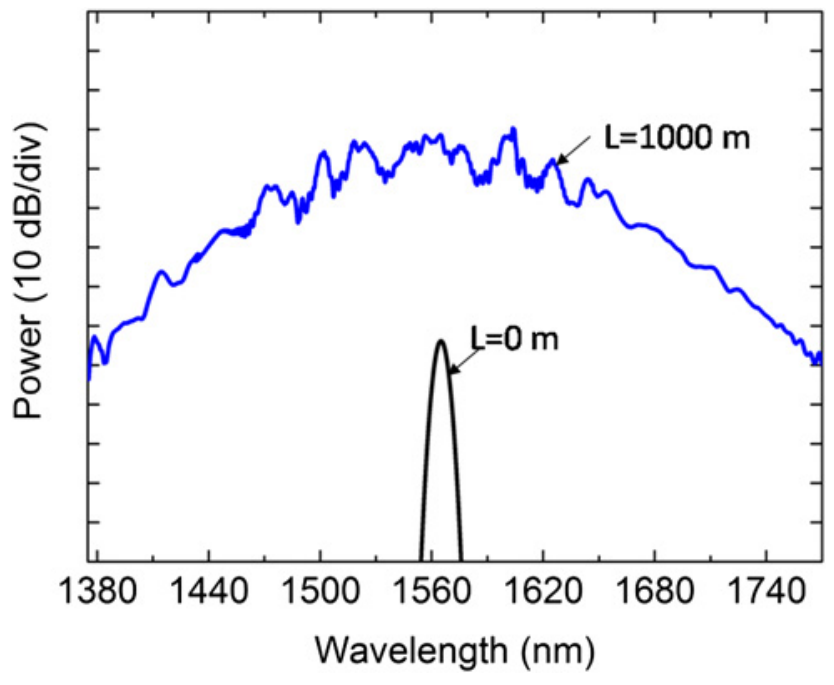

Fig. 4 Simulation results on spectral profiles of bunch of noise burst at different locations of HNLF. All spectra are offset vertically for clarification

The simulation results show that a bunch of noise burst still has a fine temporal structure along $1 \mathrm{~km}$ long HNLF. Fig. 4 plots the spectral profiles of a bunch of noise burst at different locations of HNLF. The $20 \mathrm{~dB}$ spectral bandwidth is broadened from 17 to $170 \mathrm{~nm}$ after propagation along $1 \mathrm{~km}$ HNLF. In terms of $20 \mathrm{~dB}$ spectral bandwidth and spectral shape, the simulation results are consistent with the experimental results. However, the spectral fluctuation can be observed in simulation but not observed in the experiment. This happens because in the experiment the fluctuation of spectrum is averaged out when repetitive noise bursts are launched out and detected by a slow detector [6]. The simulation results here confirm that the ASE light propagating along HNLF can be treated as a bunch of noise burst and the SC generation is originated from the interplay among fibre dispersion, non-linearity, and Raman effect.

\section{Conclusion}

In this paper, we demonstrate an incoherent light source based on RSOAs as pump for high-power SC generation. The obtained results have relatively high spectral density. The power level is about $160 \mathrm{~mW}$ and $20 \mathrm{~dB}$ spectral bandwidth is about $170 \mathrm{~nm}$. The simulation results show that pulses generated from RSOA can be treated as a bunch of noise burst and the SC generation along the HNLF is originated from the interplay among fibre dispersion, non-linearity, and Raman effect.

\section{Acknowledgment}

This work was partially supported by the Academic Research Fund Tier 2 grant (ARC26/14) of Ministry of Education (MOE), Singapore.

\section{References}

[1] Genty G., Coen S., Dudely J.M.: 'Fiber supercontinuum sources (invited)', J. Opt. Soc. Am. B, 2007, 24, (8), pp. 1771-1785

[2] Kieu K., Jones R.J., Peyghambarian N.: 'High power femtosecond source near 1 micron based on an all-fiber Er-doped mode-locked laser', Opt. Express, 2010, 18, (20), pp. 21350-21355

[3] Lim J., Knabe K., Tillman K.A., ET AL.: 'A phase-stabilized carbon nanotube fiber laser frequency comb', Opt. Express, 2009, 17, (16), pp. $14115-14120$

[4] Chow K.K.: 'High average power super-continuum generation using 1 $\mu \mathrm{m}$ noise burst and highly-nonlinear photonic crystal fibre', Electron. Lett., 2012, 48, (13), pp. 781-783 
[5] Jin A., Zhou H., Zhou X., ET AL: 'High-power ultraflat near-infrared supercontinuum generation pumped by a continuous amplified spontaneous emission source', IEEE Photonics J., 2015, 7, (2), pp. 1600409-1-1600409-10
[6] Takshima Y.: 'High average power, depolarized supercontinuum generation using a $1.55 \mu \mathrm{m}$ ASE noise source', Opt. Express, 2005, 13, (15), pp. 5871-5877

[7] Agrawal G.: 'Nonlinear fiberoptics' (Academic Press, San Diego, CA, 2001) 